\title{
Systematic differences between BNP immunoassays: Comparison of methods using standard protocols and quality control materials
}

\author{
Maria Franzini a,b,*, Silvia Masotti a , Concetta Prontera ${ }^{\mathrm{b}}$, Andrea Ripoli ${ }^{\mathrm{b}}$, Claudio Passino ${ }^{\mathrm{a}, \mathrm{b}}$, \\ Stefania Giovannini ${ }^{\mathrm{c}}$, Giancarlo Zucchelli ${ }^{\mathrm{c}}$, Aldo Clerico ${ }^{\mathrm{a}, \mathrm{b}}$ \\ a Scuola Superiore Sant'Anna, Pisa, Italy \\ b Department of Laboratory Medicine, Fondazione Toscana G. Monasterio, Pisa, Italy \\ c CNR Institute of Clinical Physiology and QualiMedLab srl, Pisa, Italy
}

\section{A R T I C L E I N F O}

\section{Article history:}

Received 28 May 2013

Received in revised form 2 July 2013

Accepted 2 July 2013

Available online 9 July 2013

\section{Keywords:}

BNP

Immunoassay

Method comparison

Quality control

Heart failure

\begin{abstract}
A B S T R A C T
Background: Recent studies suggested that there are marked systematic differences among BNP immunoassays. In this study we compared the BNP data and clinical results obtained with different immunoassays, including a new method (ST-AIA-PACK, TOSOH Corporation).

Methods: BNP was measured on plasma-EDTA samples of healthy subjects $(H S, n=126)$ and patients with heart failure (HF, $\mathrm{n}=31$ NYHA I, II; $\mathrm{n}=46$ NYHA III, IV) using the ST-AIA-PACK and the Triage Biosite (Beckman Coulter) methods. Control samples distributed in the CardioOrmoCheck external quality assessment were also measured with $\mathrm{TOSOH}$ and the most used BNP immunoassays in Italy.

Results: TOSOH method showed a good correlation $(\mathrm{R}=0.976 ; \mathrm{n}=327)$ but a mean bias $(-46.9 \%)$ compared to Triage Biosite. On the base of the results obtained in 10 samples of the CardioOrmoCheck study, TOSOH method showed a strict agreement with ADVIA Centaur, while it underestimated BNP in comparison with Triage $(-52.5 \%)$ and ARCHITECT methods (-39.4\%). The agreement of ST-AIA-PACK and Triage Biosite methods for classification of HF patients was tested using $100 \mathrm{ng} / \mathrm{L}$ of BNP; the positive agreement between methods was $65 \%$, overall agreement was $73 \%$.

Conclusions: Our results confirm that there are marked differences in measured values among commercial methods for BNP assay.
\end{abstract}

(c) 2013 Elsevier B.V. All rights reserved.

\section{Introduction}

Cardiac natriuretic peptides, which include the Atrial Natriuretic Peptide (ANP) and B-type natriuretic peptide (BNP) and their related peptides, constitute a complex family of peptide hormones produced and secreted by the human heart [1-3]. The active peptide BNP is produced by cleavage of $\mathrm{COOH}$-terminal part of the pro-hormone (pro-BNP), while the N-terminal fragments of pro-hormone, NTproBNP, are currently considered inactive [1-3].

The measurement of circulating BNP and its related-peptides is considered the best biomarker of myocardial function [1-4], and the international guidelines recommend its use both for the diagnosis of acute heart failure (HF), and risk stratification and follow-up of patients with acute or chronic HF [5-7]. Moreover, some meta-analyses $[8,9]$ confirmed that both BNP and NT-proBNP immunoassays have a high degree of diagnostic accuracy and clinical relevance in both acute and chronic heart failure.

\footnotetext{
* Corresponding author at: Scuola Superiore Sant'Anna, c/o Fondazione Toscana G. Monasterio, Via G. Moruzzi 1, 56124 Pisa, Italy. Tel.: + 39050 3153309; fax: + 39 0503152166.

E-mail addresses: franzinimaria@gmail.com, m.franzini@sssup.it (M. Franzini).
}

However, recent studies suggested that a great part of B-type natriuretic peptides measured by commercially available immunoassays in patients with cardiovascular disease is devoid of any biological activity [2,3,10-12]. Results of the CardioOrmoCheck study (i.e., a proficiency testing program, based on an external quality assessment scheme) recently demonstrated that there are significant differences in analytical characteristics and measured values among the most popular commercial methods for B-type related natriuretic peptides, especially among the immunossays considered specific for BNP $[13,14]$. In particular, more than 2 folds difference was on average found between the BNP values measured by the two methods reporting the highest and the lowest values, respectively $[13,14]$; while the NT-proBNP immunoassays showed only slightly differences in both imprecision and measured vaules. Similar results were also previously obtained by another study performed in only one laboratory [15]. However, in spite of these great differences in analytical and clinical performances, the most recent international guidelines [5-7], still suggest identical decisional values for all BNP immunoassay methods (i.e., $100 \mathrm{ng} / \mathrm{L}$ ).

This pragmatical approach is actually conceivable and shearable only if the new BNP immunoassay methods will be in agreement with the cosensus mean of the methods available from many years in the market. Indeed, this fact might suggest a trend of the "market" 
to spontaneously reach a harmonization between the BNP values measured by different methods. In order to test this hypothesis we compared with other commercially available methods the BNP data and clinical results obtained with a new BNP immunoassay (named ST-AIA-PACK BNP by TOSOH CORPORATION), recently brought into market, and for this reason not evaluated in CardioOrmoCheck studies $[13,14]$.

\section{Materials and methods}

\subsection{Collaborative study}

The CardioOrmoCheck study is a proficiency testing program for the measurement of BNP and NT-proBNP, organized in Italy since 2005 under the patronage of the Study Group of the Cardiovascular Risk Biomarkers of the Italian Society of Clinical Biochemistry (SIBIOC) by three accredited (ISO 9001 accreditation) Italian laboratories: CNR QualiMedLab and Department of Laboratory Medicine of Fondazione Toscana G. Monasterio (Pisa, Italy), Department of Laboratory Medicine, University-Hospital (Padova, Italy), and Biomedical Research Centre (Castelfranco Veneto, Italy). The detailed program and study protocol of this collaborative study have been previously described $[13,14]$. In the last cycle (year 2012), 112 Italian laboratories participated to the CardioOrmoCheck study: 43 laboratories (38\%) used BNP methods, while 69 NT-proBNP methods (62\%). From the 43 laboratories, which measured the 10 study samples with BNP methods, 515 assay results were available.

\subsection{Sample preparation for the collaborative study}

For the preparation of study samples, several EDTA plasma were pooled together to obtain a sample pool with final volume of about $100 \mathrm{~mL}$, which was immediately stored at $-20{ }^{\circ} \mathrm{C}$. All samples were tested for absence of HBsAg, antiHCV, and antiHIV. Plasma pools were prepared using residuals from samples collected from apparently healthy subjects (also divided according to gender) and patients with cardiac diseases with or without symptomatic heart failure. Blood samples collected from approximately $30-50$ subjects/patients were included in every study sample. Subjects and patients gave the informed consensus for the use of their residual blood samples in the study. Study samples were sent by mail as lyophilized materials. Lyophilization procedure was performed by Polymed (Sambuca, Firenze, Italy) within two weeks after preparation of sample pools. Stored sample pools, were defrozen, then distributed in approximately 150 vials (each containing a plasma/serum volume of $0.5 \mathrm{~mL}$ ), and finally lyophilized, as previously reported $[13,14]$. The lyophilized materials were reconstituted with $0.5 \mathrm{~mL}$ of distilled water by participant laboratories before the assay. BNP and NT-proBNP concentrations of all the study samples were measured before and after the liophilization by the reference laboratory (i.e., Laboratory of the Fondazione Regione Toscana G. Monasterio, Pisa) in order to evaluate the recovery of lyophilization procedure and the stability of BNP and NT-proBNP in the matrix samples. Samples with unreliable results were discarded. In particular, only the samples with BNP and NT-proBNP values, which showed peptide values similar to the pathophysiological characteristics of original samples (i.e., healthy subjects, patients with moderate or more severe heart failure) [1-3], were distributed as study samples in the survey.

\subsection{BNP measurements}

BNP values were measured in the reference laboratory with the novel ST-AIA-PACK BNP method (TOSOH Corporation, Tokyo, Japan) and the Triage Biosite (Beckman Coulter), which is the routine method used by the reference laboratory.
The ST-AIA-PACK BNP is a two-site immunoenzymometric assay that uses two monoclonal antibodies. Standard materials and the antibodies are supplied by the Shionogi's Diagnostic Division (personal communications from TOSOH EUROPE N.V., Tessenderlo, Belgium): the capture antibody is against the ring structure (KYhBNP-II; amino acids 14-21), the detection antibody is against COOH-terminus (BC203; amino acids 27-32) [16].

The capture antibody is immobilized on magnetic beads, while the detection antibody is labeled with alkaline phosphatase. The magnetic beads are washed to remove unbound enzyme-labeled monoclonal antibody and are then incubated with the fluorogenic substrate 4methyllumbelliferyl-phosphate. The amount of enzyme-labeled monoclonal antibody that binds to the beads is directly proportional to the BNP concentration in the test sample. The ST-AIA-PACK BNP method was performed on the AIA-2000 platform (TOSOH Corporation).

The limits of blank (LoB) and detection (LoD) and of quantitation for ST-AIA-PACK BNP method were determined according to the CLSI EP17-A protocol [17]. The calculated values of LoB and LoD were $2.6 \mathrm{ng} / \mathrm{L}$ and $5.4 \mathrm{ng} / \mathrm{L}$, respectively; LoQ at $20 \% \mathrm{CV}$ and $10 \% \mathrm{CV}$ were $9 \mathrm{ng} / \mathrm{L}$ and $30 \mathrm{ng} / \mathrm{L}$, respectively.

Triage Biosite (Beckman Coulter, Inc, Fullerton, CA 92835 USA) method was performed on the UniCell DxI 800 platform. Analytical performance of the Triage Biosite immunoassay for BNP measurement had been previously tested in the reference laboratory and reported elsewhere [18].

\subsection{Experimental patients and samples}

To evaluate and compare the analytical characteristics and clinical results of the ST-AIA-PACK and Triage Biosite BNP methods, patients with stable chronic heart failure, in follow up at the Fondazione Toscana G. Monasterio, were enrolled in this study. The diagnosis of heart failure was made according to the international guidelines $[5,6]$. Cardiac morphology and function were assessed by 2D echocardiography, or cardiac catheterization, when needed, as previously described [19]. Patients were stratified according to the New York Heart Association (NYHA) functional classification system [19]. In order to reduce the blood volume needed for the study and the discomfort for the patients, BNP was measured from the residuals of specimens obtained as standard clinical practice, and no additional samples were obtained. All healthy people were enrolled from the laboratory staff, blood donors, or voluntary subjects, included in screening programs for preventive medicine. The informed consent was obtained by all subjects and patients enrolled in the study.

Blood samples were collected in polypropylene tubes containing EDTA $(1 \mathrm{mg} / \mathrm{mL})$ and aprotinine $(500 \mathrm{kIU} / \mathrm{mL})$ in the reference laboratory (i.e., Laboratory of the Fondazione Toscana G. Monasterio, Pisa, Italy). Then, samples were rapidly centrifuged at $3000 \times \mathrm{g}$ for $10 \mathrm{mi}-$ nutes and plasma was analyzed as soon as possible. If it was not possible to perform the assay within one hour, the samples were stored at $-20^{\circ}$ $\mathrm{C}$ until the assay.

\subsection{Statistcal analysis}

Standard statistical analyses were carried on by a Macintosh Dual $2.3 \mathrm{GHz}$ PowerPC G5 using the Stat-View 5.0.1 program (1992-98, SAS Institute Inc., SAS Campus Drive, Cary, NC, USA).

The agreement between BNP values measured with ST-AIA-Pack and Triage Biosite was analysed by Bland-Altman plot. The comparison of BNP values among healthy subjects, patients with moderate (functional NYHA class I and II) or severe heart failure (NYHA class III and IV) were performed by post hoc Schiffè test after 1way-ANOVA using log transformate of original values, because the BNP values did not show a normal distribution, but an approximated log-normal distribution. Pearson analysis has been performed to assess the correlation between log-transformed BNP values and left ventricular ejection 
fraction (LVEF). The classification agreement between ST-AIA-PACK and Triage Biosite methods was calculated using $100 \mathrm{ng} / \mathrm{L}$ of BNP as cut-off. The overall agreement corresponded to the proportion of cases for which the two BNP methods agree. The positive agreement between methods was calculated dividing the number of patients with BNP values over the cut-off measured with ST-AIA-PACK by those measured with Triage Biosite method.

\section{Results}

\subsection{Comparison between ST-AIA-PACK and Triage Biosite BNP methods}

In the reference laboratory, a very close linear relationship was found between the BNP values obtained measuring 327 EDTA-plasma samples of healthy subjects and cardiac patients with ST-AIA-PACK BNP and BNP Triage Biosite methods (Fig. 1A). However the ST-AIA-PACK BNP method showed a significant negative bias (i.e., underestimation) of BNP values compared to Triage Biosite method equal to $-46.9 \% \pm$ $23.4 \%$ (mean \pm SD, $\mathrm{p}<0.0001$ ) (Fig. 1B). No significant correlation

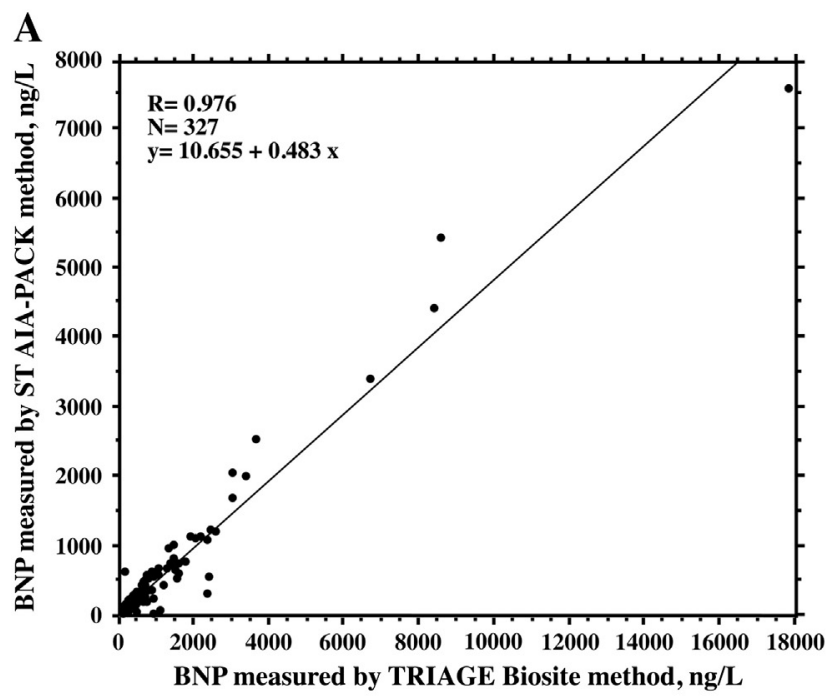

B

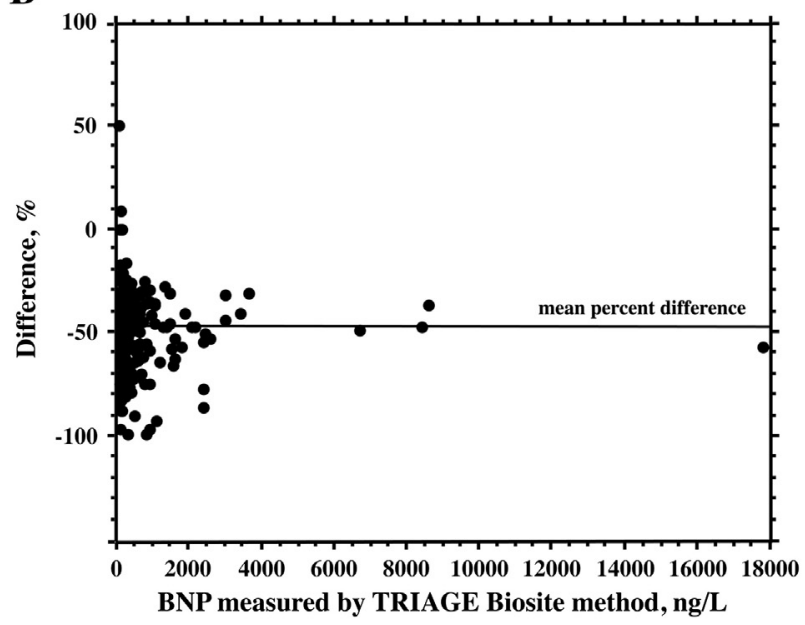

Fig. 1. Comparison between ST-AIA-PACK and Triage Biosite methods. Panel A. Linear regression found measuring 327 EDTA-plasma samples of healthy subjects and cardiac patients with ST-AIA-PACK BNP method, using the AIA-2000 platform (Y-axis) and BNP Triage Biosite (Beckman Coulter, Inc, Fullerton, CA 92835 USA) method using the UniCell DxI 800 platform (Y-axis). Panel B. Scatchard plot between the percent difference values between the BNP values obtained with ST-AIA-PACK BNP and BNP Triage Biosite methods [(ST-AIA-PACK - Triage Biosite)/Triage Biosite x 100, Y-axis] and the BNP concentration measured by BNP Triage Biosite method (X-axis). The continuous line refers to the calculated mean difference. was found between the percentage difference [(ST-AIA-PACK - Triage Biosite)/Triage Biosite $\mathrm{x} 100, \mathrm{Y}$-axis] and the BNP concentration measured by the BNP Triage method $(R=0.033, p=0.5512)$, while a close correlation was found between the absolute difference of the values obtained with the two methods and the measured BNP concentrations with the Triage method $(\mathrm{R}=0.970, \mathrm{p}<0.0001)$.

The 10 study samples of the CardioOrmoCheck study, distributed in the 2012 annual cycle, were also measured by BNP ST-AIA-PACK in the reference laboratory in order to allow a comparison with the most used BNP methods in Italy (Triage Biosite by Beckman Coulter, ARCHITECT by Abbott Diagnostics and ADVIA Centaur by Siemens Health Care) (Fig. 2). These data showed a close correlation between the BNP values repeatedly $(\mathrm{n}=10)$ measured with the ST-AIA-PACK
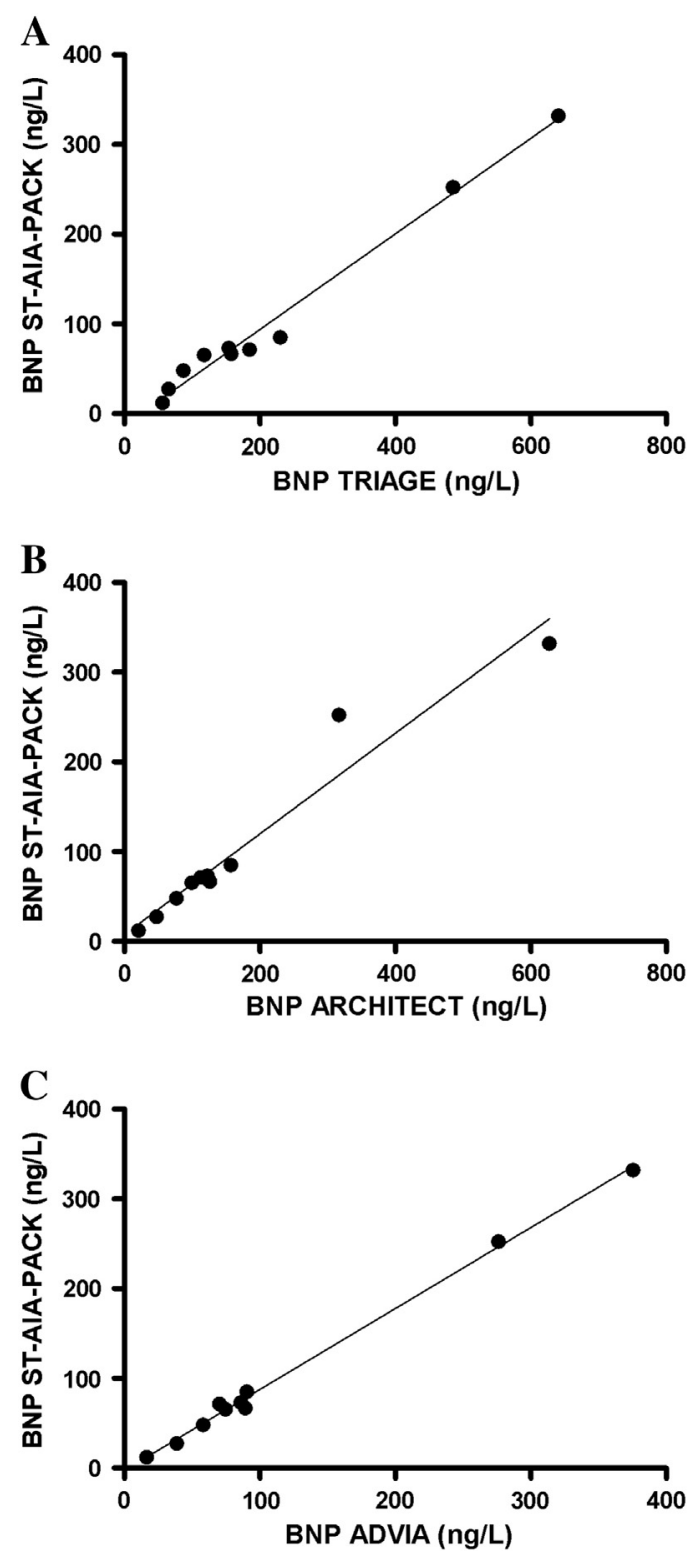

Fig. 2. Regressions between BNP methods using the study samples of CardioOrmocheck study. Linear regressions analysis between BNP values repeatedly $(n=10)$ measured with ST-AIA-PACK method by the reference laboratory and respective consensus mean values found in the 10 study samples measured with other three methods by all laboratories participating to the CardioOrmoCheck study. Panel A: Regression between ST-AIA-PACK (Y-axis) and the BNP Triage Biosite method (Y-axis); $Y=-12.7+0.5327$ $\mathrm{X} ; \mathrm{R}=0.9926, \mathrm{~N}=10$. Panel B: Regression between ST-AIA-PACK (Y-axis) and the ARCHITECT method (Y-axis); $\mathrm{Y}=7.710+0.5609 \mathrm{X} ; \mathrm{R}=0.9702, \mathrm{~N}=10$. Panel C: Regression between ST-AIA-PACK (Y-axis) and the ADVIA Centuar method (Y-axis); $\mathrm{Y}=-2.585+0.9023 \mathrm{X} ; \mathrm{R}=0.9981, \mathrm{~N}=10$. 
method (Y-axis) by the reference laboratory and respective consensus mean values found with the other three methods (X-axis) by all laboratories participating to the CardioOrmoCheck study. In particular, ST-AIA-PACK method showed a good agreement with ADVIA Centaur method (mean difference $-12.0 \%$ ) (Fig. 2C), while it greatly underestimated BNP values in comparison with both BNP Triage Biosite (mean difference $-52.5 \%$ ) (Fig. 2A) and ARCHITECT methods (mean difference $-39.4 \%$ ) (Fig. 2B).

\subsection{Clinical results}

We measured the plasma concentration in 126 healthy subjects [ 72 males; age, mean (SD), 49.3 (11.9) years], and 76 patients with chronic heart failure: 31 patients with moderate heart failure [functional NYHA class I and II; 19 males; age 54.9 (10.1) years; LVEF 40.3\% (12.5)] and 46 patients with severe heart failure [functional NYHA class III and IV; 19 males; age 74.3 (10.9) years; LVEF 33.1\% (9.5)]. Highly significant differences $(\mathrm{p}<0.0001)$ were found between the mean BNP values found in the 3 subgroups measured with ST-AIA-PACK (Fig. 3A) or with Triage Biosite (Fig. 3B). Log-transformed BNP values obtained with both methods showed a negative correlation with LVEF, with similar correlation coefficients [ST-AIA-PACK: $r=-0.558$ (95\%CI: -0.696 ; -0.380 ), $\mathrm{p}<0.0001$; Triage Biosite: $\mathrm{r}=-0.545$ (95\%Cl: -0.686 ; $-0.365)]$.

The agreement of ST-AIA-PACK and Triage Biosite methods for classification of HF patients was tested using $100 \mathrm{ng} / \mathrm{L}$ of BNP, which is the cut-off value suggested by international guidelines [5,7]: 17 and 39 out of 77 patients showed concordant BNP values $<$ or $>100 \mathrm{ng} / \mathrm{L}$, respectively, with both methods; 21 patients showed BNP values higher than $100 \mathrm{ng} / \mathrm{L}$ with Triage Biosite but lower with ST-AIA-PACK; no patients showed BNP values higher than the cut-off with ST-AIA-PACK but lower with Triage Biosite. Thus overall agreement was 73\% and positive agreement (>100 ng/L) was $65 \%$.

\section{Discussion}

Our results confirm that large systematic differences are present between the most popular BNP immunoassays. Indeed, wide differences between the results of BNP immunoassays had been also previously reported [13-15,20]. In particular, several studies demonstrated that the ADVIA method for Centaur platform (by Siemens Health Care Diagnostics) and the IRMA method (by Shionogi's Diagnostic Division, Japan) measured lower (up to the half) BNP values in comparison with other immunoassays, such as the POCT Triage method (Alere Diagnostics), the BNP Triage Biosite for Access and UniCell DxI platforms (Beckam Coulter Diagnostics), the MEIA method for AxSYM system, and the chemiluminescent microparticle immunoassay for ARCHITECT platform (Abbotts Diagnostics) $[13,14,20]$. The present study demonstrates that also the ST-AIA-PACK method for the AIA platform, only very recently brought into market, present BNP values that are about the half compared to other very popular BNP immunoassays, such as the BNP Triage Biosite for UniCell DxI platform and the BNP method for ARCHITECT platform (Figs. 2 and 3). On the other hand, this new ST-AIA-PACK method shows BNP values similar to those found by laboratories using the ADVIA method for Centaur platform in the CardioOrmoCheck study (Fig. 2C). It is interesting to note that the ST-AIA-PACK method uses the same antibodies and standard materials of IRMA Shionogi and ADVIA method for Centaur platform, supplied by the Shionogi's Diagnostic Division.

In conclusions, our data confirm that there are marked differences in analytical performance and measured values especially among commercial methods for BNP assay. These findings suggest that may be not reasonable to suggest identical cut-off or decision values for all BNP immunoassays, as recommended by international guidelines [5-7]. These data also suggest that clinicians should be carefull
A

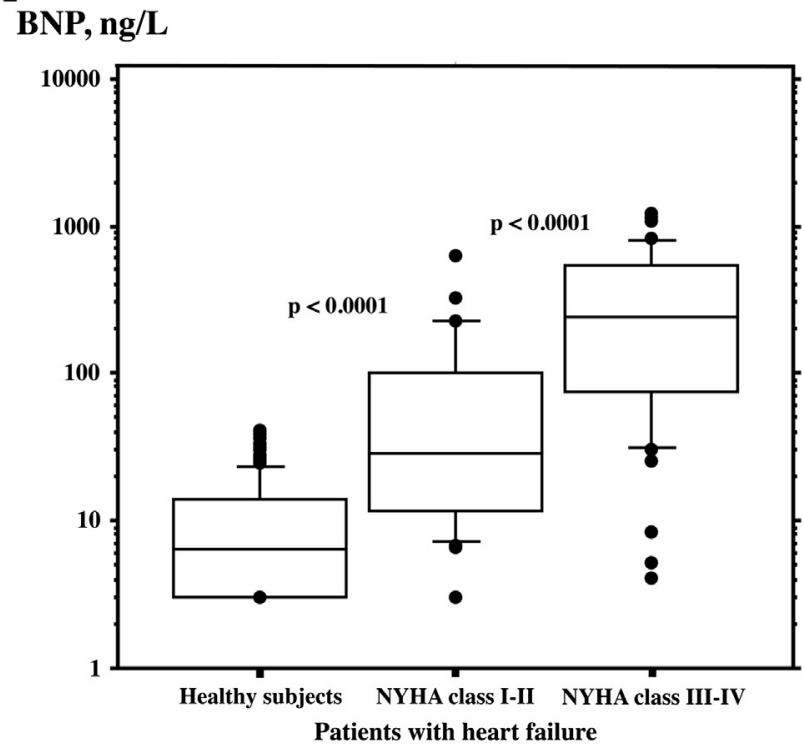

B

BNP, ng/L

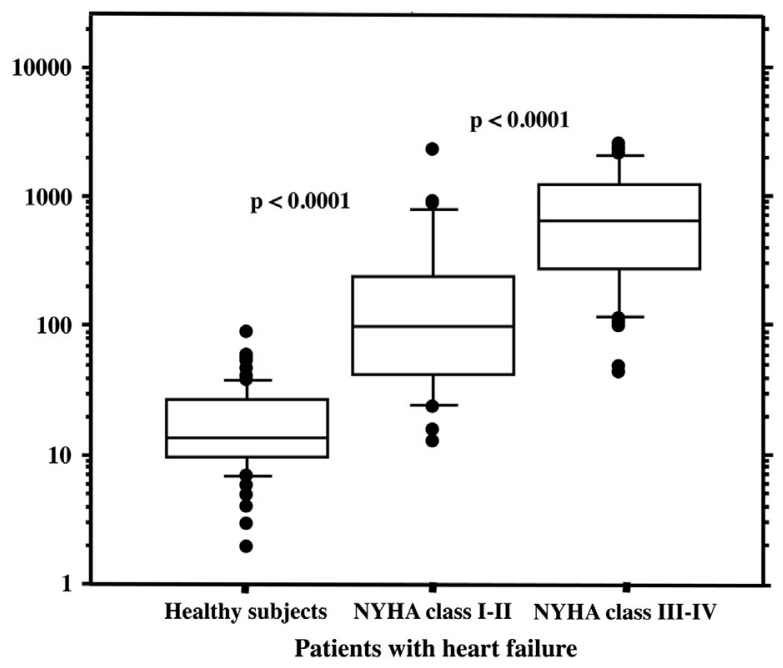

Fig. 3. Distribution of BNP values in healthy subjects and patients with heart failure Distribition of BNP values measured with the ST-AIA-PACK method (Panel A) and Triage Biosite method (Panel B) in 125 healthy subjects and patients with moderate (functional NYHA class I - II, $\mathrm{n}=31$ ) or severe (functional NYHA classes III - IV, $\mathrm{n}=46$ ) heart failure. The data are reported as boxes indicating the 25th, 50th (median), 75th percentiles of BNP values, the whiskers correspond to the 10th, and 90th percentiles; the outliers were indicated as separated black circles. The concentractions (Y-axis) are reported as log-scale. The levels of statistical significance ( $\mathrm{p}$ values) are also indicated in the figure.

when comparing results obtained from different laboratories, which use different BNP assays.

\section{Sponsorship}

TOSOH EUROPE N.V. (Tessenderlo, Belgium) kindly supplied all reagents and calibrators used in the study for the measurement of BNP with AIA-2000 platform.

\section{References}

[1] Clerico A. Pathophysiological and clinical relevance of circulating levels of cardiac natriuretic hormones: Are they merely markers of cardiac disease? Clin Chem Lab Med 2002;40:752-60. 
[2] Clerico A, Giannoni A, Vittorini S, Passino C. Thirty years of the heart as an endocrine organ: physiological role and clinical utility of cardiac natriuretic hormones. Am J Physiol Heart Circ Physiol 2011;301:H12-20.

[3] Clerico A, Vittorini S, Passino C. Measurement of the pro-hormone of brain type natriuretic peptide (proBNP): methodological considerations and pathophysiological relevance. Clin Chem Lab Med 2011;4:1949-54.

[4] Tang WH, Francis GS, Morrow DA, et al. National Academy of Clinical Biochemistry Laboratory Medicine practicNICEe guidelines: Clinical utilization of cardiac biomarker testing in heart failure. Circulation 2007;116:e99-109.

[5] Dickestein K, Cohen-Solal A, Filippatos G, et al. The Task Force for the Diagnosis and Treatment of Acute and Chronic Heart Failure of the European Society of Cardiology. ESC Guidelines for the diagnosis and treatment of acute and chronic heart failure 2008. Eur J Heart Fail 2008;10:993-89.

[6] Yancy CW, Jessup M, Bozkurt B, et al. ACCF/AHA Guideline for the Management of Heart Failure. J Am Coll Cardiol 2013. http://dx.doi.org/10.1016/j.jacc.2013.05.019.

[7] NICE, cinical guideline no 108. Chronic heart failure. National clinical guideline for diagnosis and management in primary and secondary care; August 2010 1-222.

[8] Clerico A, Fontana M, Zyw L, et al. Comparison of the Diagnostic Accuracy of Brain Natriuretic Peptide (BNP) and the N-Terminal Part of the Propeptide of BNP Immunoassays in Chronic and Acute Heart Failure: A Systematic Review. Clin Chem 2007;53:813-22.

[9] Ewald B, Ewald D, Thakkinstian A, Attia J. Meta-analysis of B type natriuretic peptide and $\mathrm{N}$-terminal pro $\mathrm{B}$ natriuretic peptide in the diagnosis of clinical heart failure and population screening for left ventricular systolic dysfunction. Intern Med J 2008;38:101-13.

[10] Liang F, O'Rear J, Schellenberger U, et al. Evidence for functional heterogeneity of circulating B-type natriuretic peptide. J Am Coll Cardiol 2007;49:1071-8.
[11] Seferian KR, Tamm NN, Semenov AG, et al. The brain natriuretic peptide (BNP) precursor is the major immunoreactive form of BNP in patients with heart failure. Clin Chem 2007;53:866-73.

[12] Hammerer-Lercher A, Halfinger B, Sarg B, et al. Analysis of circulating forms of proBNP and NT-proBNP in patients with severe heart failure. Clin Chem 2008;54:858-65.

[13] Prontera C, Zaninotto M, Giovannini S, et al. Proficiency testing project for brain natriuretic peptide (BNP) and the N-terminal part of the propeptide of BNP (NT-proBNP) immunoassays: the CardioOrmoCheck study. Clin Chem Lab Med 2009; $47: 762-8$

[14] Clerico A, Zaninotto M, Prontera C, et al. State of the art of BNP and NT-proBNP immunoassays: The CardioOrmoCheck study. Clin Chim Acta 2012:414:112-9.

[15] Rawlins ML, Owen WE, Roberts WL. Performance characteristics of four automated natriuretic peptide assays. Am J Clin Pathol 2005;123:439-45.

[16] Apple FS, Panteghini M, Ravkilde J, et al. Quality specifications for B-type natriuretic peptide assays. Clin Chem 2005;51:486-93.

[17] CLSI EP17-A protocol. Protocols for determination of limits of detection and limits of quantitation; approved guidelines, vol. 24, No. 34; 2004 [Wayne, Pennsylvania].

[18] Prontera C, Storti S, Emdin M, et al. Comparison of a Fully Automated Immuno assay with a Point-of-Care Testing Method for B-Type Natriuretic Peptide. Clin Chem 2005;51:1274-6.

[19] Emdin M, Passino C, Prontera C, et al. Comparison of Brain Natriuretic Peptide (BNP) and amino-terminal proBNP for early diagnosis of heart failure. Clin Chem 2007;53: 1289-97.

[20] Clerico A, Prontera C, Emdin M, et al. Analytical performance and diagnostic accuracy of immunometric assays for the measurement of plasma BNP and NT-proBNP concentrations. Clin Chem 2005;51:445-7. 